\title{
CSMA/SF: Carrier Sense Multiple Access with Shortest First
}

\author{
Guanhua Wang, Student Member, IEEE, Kaishun Wu, Member, IEEE, and Lionel M. Ni, Fellow, IEEE
}

\begin{abstract}
Energy efficiency is the main concern in wireless sensor networks (WSNs) due to devices' limited battery power. Because the heavy burden of nodes that near the sink, this "energy hole problem" makes nodes near the sink have faster energy depletion than others. Because of this, the lifetime of WSNs, to some extent, is determined by the power consumption of communication between sink and sensing nodes that near the sink. To address this issue, we propose CSMA/SF (Shortest First) protocol to reduce power consumption of sink-node communication by minimizing energy cost in carrier sense during nodes' channel contention. CSMA/SF modifies existed CSMA/CA MAC protocol. Instead of complete contention-based, CSMA/SF ensures nodes remaining shorter message has higher priority in contention by implementing a distributed scheduling algorithm and incorporating Length Detection scheme. Further, CSMA/SF employs an Anti-Starvation mechanism to solve the starvation problem of shortest-first protocol. CSMA/SF also optimizes channel utilization by reducing the probability of collisions. We have implemented CSMA/SF into USRP2 platform and also conducted comprehensive simulations. The experimental results show that CSMA/SF can reduce overall energy consumption by around $20 \%$. CSMA/SF can improve channel utilization up to $40 \%$.
\end{abstract}

Index Terms-Energy efficiency, CSMA, MAC.

\section{INTRODUCTION}

W IRELESS sensor networks (WSNs) are widely deployed in many kinds of applications. There is a wide range of WSNs implementation in environmental surveillance, robotic exploration, health monitoring and so forth. Despite the huge variety of WSN's potential utilizations, the need for aggressive power saving is always the primitive issue. It is because these simple sensors are intended to operate without recharging for a long period of time.

Because of the communication in wireless sensor networks has many-to-one property, the nearer to the sink, the heavier workload the nodes will have. Generally, a typical WSN contains many sensor nodes and one or more sinks. The sensor nodes are used to sense and collect information from environment. And the collected data will be delivered to the sink. However, the data collected from all nodes are transmitted through multi-hop routing will be concentrated to a

Manuscript received August 7, 2013; revised December 19, 2013; accepted January 1,2014 . The associate editor coordinating the review of this paper and approving it for publication was T. Hou.

$\mathrm{K}$. Wu is with the College of Computer Science and Software Engineering, Shenzhen University. He is also with Guangzhou HKUST Fok Ying Tung Research Institute (e-mail: kwinson@ust.hk).

G. Wang and L. Ni are with the Department of Computer Science and Engineering, Hong Kong University of Science and Technology, Clear Water Bay, Kowloon, Hong Kong e-mail: \{gwangab, ni\}@cse.ust.hk.

Digital Object Identifier 10.1109/TWC.2014.020414.131457 few receivers (i.e. sinks). The sensor nodes that around the sink will need to relay data that collected from outer-ring nodes which faraway from the sink. Thus, the sensor nodes near the sink will have faster power depletion than the outer-ring nodes. This phenomenon is called "energy hole problem"[15] [16] [29] or "crowded center effect"[23].

There are many papers lengthen WSNs lifetime by addressing the "energy hole problem" [23] [15] [32] [16] [29]. In [15] [16], they propose a formal mathematical model for analysing "energy hole problem" in WSNs. Some other approach [23] replaces shortest path routing to curveball routing to mitigate "crowded center effect". The authors in [29] leverages nonuniform node distribution whereas [32] scheme reduces energy consumption in mobile sink scenario to mitigate this problem.

To the best of our knowledge, none of previous works have ever focused on energy consumption of carrier sense when sensor nodes contend for channel access to transmit data to sink. This is because the initial purpose of carrier sense is to reduce energy cost and collision [19] [22]. Nevertheless, when deploying large-scale WSNs, the energy cost of carrier sense during nodes-sink communication cannot be negligible. Several papers [22] [14] claim that carrier sense in nodes-sink communication is one of the key energy cost, which may equal to energy cost of receiving or transmitting.

This paper presents CSMA/SF (Carrier Sense Multiple Access with $\underline{\text { Shortest }}$ First), a new MAC protocol designed for WSNs to lengthen their operation lifetime. The goal of CSMA/SF is also to remedy the "energy hole problem". Different from previous approaches, we reduce energy consumption between sink-node communication in another way. That is to minimize the energy cost of carrier sense process during many-to-one data transmission between nodes and sink. To address this, we modify the purely contention-based CSMA/CA MAC protocol to have the attribute of contention priority.

The key idea of CSMA/SF is to let nodes with shorter data first finish their transmission so that they do not need to continuously sense the channel any longer. By doing this, it reduces the energy cost of these nodes and minimizes the WSN's overall energy cost of carrier sense. Nevertheless, how to realize it is a non-trivial problem. We implement a distributed shortest-first scheduling algorithm. We incorporate Length Detection scheme to determine which node remains the shortest data to transmit and let it has the highest priority in channel contention.

For the ease of understanding, we illustrate a simple example of CSMA/SF in Fig.1. Suppose node1 needs to transmit 1 


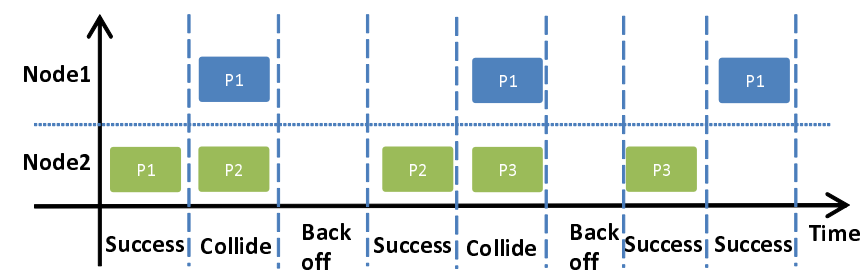

(a) Channel Contention in CSMA/CA.

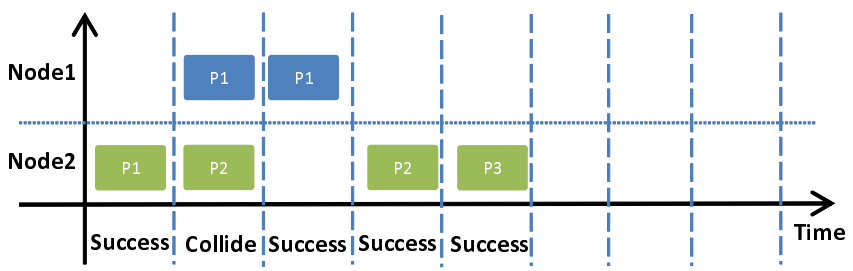

(b) Channel Contention in CSMA/SF

Fig. 1. Comparison of channel access in CSMA/CA and CSMA/SF MAC protocol.

packet whereas node 2 for 3 packets. Because of CSMA/CA's completely contention-based character, there may have high probability of collision. When collision happens, CSMA/CA uses randomly back-off mechanism to reduce probability of collision happens again. In CSMA/CA, the total carrier sense rounds are 5 of node 1 (i.e. time slot $2,4,5,7,8$ ) plus 5 of node2 (i.e. time slot $1,2,4,5,7$ ), which is equal to 10 . Whereas in CSMA/SF, it ensures nodes with less data has higher priority in transmission. It needs only 6 sensing round in total (i.e. for node1 needs to sense 2,3 slot whereas node2 needs to sense 1,2,4,5 slot). By implementing CSMA/SF can apparently reduce energy cost for carrier sense.

CSMA/SF can not only save energy in carrier sense, but also improve channel utilization compared with traditional CSMA/CA protocol. As in Fig.1, finishing the whole transmission in CSMA/CA costs 8 time slots whereas in CSMA/SF it only needs 5 . The reason for this is that by implementing shortest-first scheduling algorithm, we reduce the time of control procedure in medium access control (e.g. time wasted in randomly back-off). More precisely, because of CSMA/SF is priority-based instead of complete contention-based ones (e.g. CSMA/CA), it reduces the probability of collision. Less collision means less control time wasted. This control time reduction will improve channel utilization. We give a formal mathematical analysis of CSMA/SF's channel utilization efficiency in section 3.

Nevertheless, CSMA/SF also introduce some problems and overhead. First, by implementing shortest first scheduling algorithm, it will cause starvation problem. More precisely, there may exists the chance that nodes with long data to transmit cannot get access to channel for long time. To address this, we design Anti-Starvation mechanism which let nodes with long data get chance to send it to the sink periodically. And this periodical data transmission will decrease the length of long data, which will solve the starvation problem. Another overhead may occur in implementing Length Detection scheme. In order to know current transmitting node's remaining data length, the listening nodes need to synchronize with the transmitting node for their carrier sense time slot. In other word, the carrier sensing nodes need to listen to the whole synchronization header [13] of transmitting node. However, the part is very small and can be negligible. For example, in IEEE 802.15.4 [13] standard, the preamble is only 10 symbols in $2.4 \mathrm{GHz}$ band. Compared with the benefits that CSMA/SF can achieve, this trade-off is worth being made.

To sum up, the main contributions of this work are summarized as follows:

- CSMA/SF is the first to propose energy saving scheme in carrier sense of node-sink communication. We achieve this by implementing shortest-first scheduling algorithm that allow nodes with short data first finish their transmission.

- CSMA/SF incorporates Length Detection scheme to determine which node remains the shortest data to transmit.

- Anti-Starvation mechanism is designed for solving the starvation problem of shortest-first scheduling protocol. The primitive is to let nodes with long data periodically transmit it to sink in order to reduce the length of data.

- We implemented CSMA/SF on USRP2 platform and simulated this protocol on NS2 for large-scale WSN scenarios. Results shows that, compared with CSMA/CA, our protocol can reduce energy cost of carrier sense around $37 \%$, which is around $20 \%$ overall energy cost with nodes number above 10 . We also improve channel utilization up to $40 \%$.

The rest of this paper is organized as follows. Section 2 surveys the related work and describes our motivation. In section 3, we first give some preliminaries and then deliver detailed strategy of CSMA/SF protocol with theoretical analysis. We implemented CSMA/SF in USRP2 platform [7] and also simulated it in NS2 simulator [20] in Section 4. Evaluation of CSMA/SF is described in Section 5. Section 6 concludes the paper.

\section{Related Work AND Motivation}

\section{A. Related Work}

Energy efficiency in the "energy hole problem" has been a hot research topic for a long time. The most related works to our CSMA/SF approach are [17] [35]. They address the "energy hole problem" by scheduling duty cycling of sensors, in order to achieve energy fairness and efficiency in WSNs. However, they are not specially designed for energy saving in the process of carrier sense, thus they cannot achieve nearly optimal energy saving in this process. Further, these approaches indeed cannot reduce the probability of collisions, whereas CSMA/SF does. Other previous works can be divided broadly in following three categories.

The first category is based on the preassumption that the sink has mobility [32] [10] [9] [25] [11] [34]. They leverage this mobility to reduce energy cost in sink-node transmission. To be more specific, In [32] [34] and [11], the authors leverage sink's mobility with another assumption that transmission is delay-tolerant. The basic idea is to let nodes hold data until sink is relatively close to it. Then the nodes send data in low power in order to save energy. The approach in [10] is focusing on dynamically finding the minimum energy cost multi-hop route for each node's data relay. [9] and [25] are proposed to 
determine new location for sink with the purpose of energy conservation.

The second category is with static sink model [3] [4] [6] [21]. The authors in [3] [4] mainly focus on building proper routine that can achieve energy efficiency in multi-hop transmission. PEDAMACS in [6] is a extension of single-hop Time Division Multiple Access (TDMA) to multi-hop, which achieve the goal of being energy-efficient. The approach in [21] is a fair MAC protocol used for sink-node transmission with tight upper and lower performance bound.

The third kind is to introduce periodic sleep-action schedules for energy efficiency [28] [31] [18] [26]. These approaches are not limited to save energy of sink-node transmission. They can be utilized through out all the sinks and nodes in WSNs. By implementing this periodic sleep-action method, it can save the amount of energy as large proportion as the ratio of sleep mode to wake-up mode. However, if this periodic sleep mode does not scheduled well, it will cause multi-hop transmission suspended because of the nodes with inappropriate sleeping cycle.

\section{B. Motivation of CSMA/SF}

Due to the "energy hole problem" in WSNs, the lifetime of WSNs can be determined by the nodes that first exhaust their energy. And those nodes first exhaust energy are apparently the nodes that around the sink. However, even though the nodes near sink deplete all their energy, other nodes still have plenty of energy. It results the lifetime of one wireless sensor network can be lengthened by make energy consumption of nodes around the sink more efficient. To be more specific, we can modify existed medium access control (MAC) to enable energy efficient communication between sink and sensor nodes near the sink. Thus, the lifetime of wireless sensor network can be lengthened.

Since there are many kinds of MAC for WSNs (e.g. TDMA, ALOHA), CSMA/CA is widely deployed for the following reasons. First, it does not need nodes to form clusters at first, where as TDMA, FDMA do. Thus the scalability of CSMA/CA is better. Second, it has overall less collisions than other contention-based protocols like ALOHA [30].

Given the related works summarized in Section 2.1, nearly none of previous works ever focus on the energy cost of carrier sense in CSMA/CA. The reason why they do not try to reduce energy cost in carrier sense is because the initial goal of implementing carrier sense is to reduce energy cost during transmission [19] [22]. To be more specific, carrier sense can reduce the probability of collision among node which concurrently transmit data to sink. The energy that carrier sense can save is the part which wasted in collision [5] [27].

Another benefit of implementing carrier sense is to improve throughput of wireless communication [33] [12]. Basically, they all leverage collision reduction property of carrier sense to improve channel utilization. In [2] paper, it analysis the performance of carrier sense and conclude that the performance can achieve nearly optimal with rate adaptation.

As carrier sense can be very beneficial to wireless communications, we cannot directly abandon it because of its energyconsumption. On the contrary, the energy cost of carrier sense among nodes cannot be negligible. In [22] [14], the authors claim that carrier sensing's energy cost can be as much as energy for transmitting or receiving. Instead of completely abandon traditional CSMA/CA, we can modify it in order to reduce energy cost of carrier sense. To achieve this, we use some other scheduling algorithm to replace completely contention-based CSMA/CA MAC protocol. Specifically, we artificially adding priority to each transmission to achieve the purpose of energy minimizing in carrier sense.

However, how to change CSMA/CA protocol with additional priority is non-trivial. As it is well-known that CSMA/CA is a FIFO (First In First Out)-like MAC protocol. It means that node which first get access to the channel transmit first. And traditionally, the basic scheduling algorithm consists of Round-Robin, Shortest-First and FIFO. Round-Robin and FIFO are both fair scheduling algorithm. In other words, these two algorithms do not have the priority attribute. And this is one of the reason why we choose Shortest-First scheduling algorithm. Another reason for using this is that only by implementing shortest-first algorithm can we minimize carrier sense's energy consumption. Based on these two main reasons, we modify CSMA/CA's FIFO-like scheduling into shortest first protocol.

In addition, how to achieve MAC protocol with priority in distributed scenario is more challenging. CSMA/CA's FIFO scheduling algorithm can be directly implemented into distributed scenario. However, in general, adding priority to each transmission should have a centralised controller to determine which one should have the highest priority. Nevertheless, in WSNs, the "Energy hole problem" itself is caused by the centralization property of the sink. If we use the sink to schedule sensor nodes around it, it will apparently introduce more overhead. Given this reason, we need to design a distributed shortest-first MAC protocol without the centralized controller (i.e. sink). And this is the reason why we need to propose a distributed shortest-first scheduling algorithm with the assistance of Length Detection scheme which broadcasts the transmitting node's remain data length.

Another problem we need to fix with is that implementing shortest-first scheduling algorithm will cause starvation problem. To address this side-effect, we design the Anti-Starvation mechanism. The basic idea of Anti-Starvation is to reduce the data length of nodes remaining long data by letting them periodically transmit their data. And this mechanism can solve starvation problem by periodically reducing the data length of nodes with heavy workload.

Another point worth mentioning is that CSMA/SF is not explicitly designed for WSNs. Any star-like topology wireless local area networks (WLANs) which need to achieve aggregate energy efficiency can deploy CSMA/SF. For example, in Olympic events, a group of many cameras deliver data to the same access point simultaneously. It can use CSMA/SF to reduce this group's overall energy cost of carrier sense during channel contention in WLANs. In order to achieve overall energy minimizing in carrier sense, each node must obey the basic rule that one do not falsify the data length it remains to transmit. 

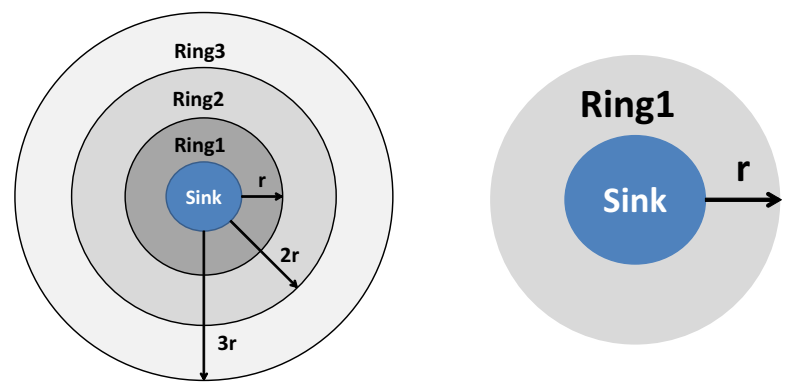

(a) Basic model for analysing "En- (b) Simplified Model in CSMA/SF ergy Hole Problem" in WSN. with randomly distributed nodes in ring 1 .

Fig. 2. Two models represent "Energy Hole Problem" around the sink.

\section{CSMA/SF PROTOCOL DESIGN}

In this section, we will deliver a detailed description about CSMA/SF MAC protocol. The main goal of our MAC protocol is to reduce energy consumption of carrier sense in sinknode communication, while supporting good channel utilization. Our protocol tries to save energy mainly from carrier sense process during nodes' channel contention. As a byproduct, CSMA/SF's shortest-first scheduling algorithm can also reduce control overhead such as back-off time and so forth. To achieve this goal, we have designed CSMA/SF that consists of three main components, namely, distributed shortest-first algorithm, Length Detection scheme and AntiStarvation mechanism.

Before we describe about these components, we first discuss our network model and some basic assumptions. After that, we deliver the detailed description about CSMA/SF's three components. Then we formally analysis our CSMA/SF with mathematic model.

\section{A. Network Model and Assumptions}

In this section, we mainly discuss about two aspects. First, we describe about classic network model of "Energy Hole Problem" in sink-node communication. And then we deliver some basic assumptions in our CSMA/SF model.

\section{A1. Network Model}

In WSN, the energy-efficiency of sink-node communication is a hot research area for many years. As described in Section 1 , sensor nodes are used for collecting information in the environment. The sink is used to gather data sensed by nodes. Usually, the relationship of node to sink is a many-to-one relationship. The basic model of this communication process can be delivered as follows.

Typically, there is one sink and many sensor nodes surround it. Based on the distance between sensors and sink, we divided the sensor nodes into several adjacent areas. For the basic network model of "Energy Hole Problem", we assume that nodes are deployed in a circular area, which is as the same in [15] [16] [29]. The only sink is located in the center of this circular area. As shown in Fig.2(a), we divide the whole circular area into several coronas based on the distance between sensor nodes and the $\operatorname{sink}(i . e . r, 2 r, 3 r$ in Fig.2).

With this basic model in mind, there are mainly two ways for energy-saving. The first one is to modify routing protocol

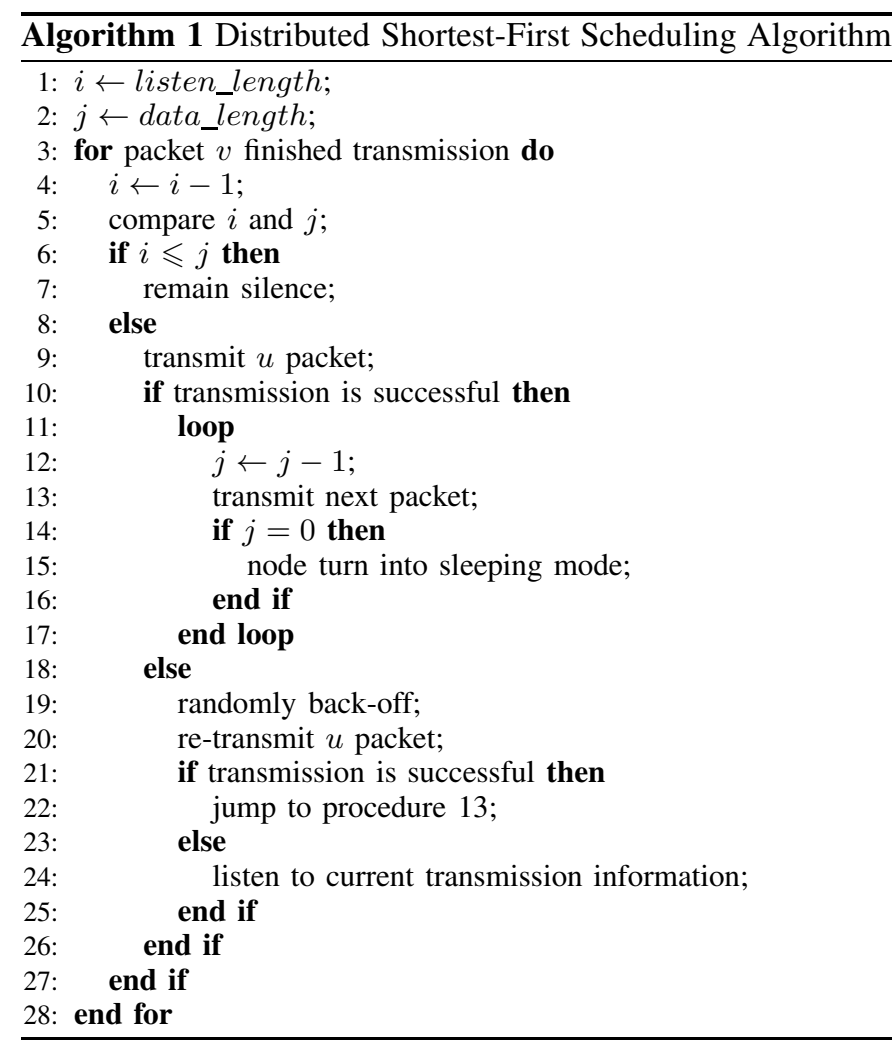

in order to balance the energy consumption of the nodes around the sink [23]. The basic idea is to change network topology which aims to give nodes that remain more energy more workload and vice versa. Indeed, this kind of method does not really reduce energy cost, it lengthens the lifetime of WSN only by balancing the workload. The second scheme is to reduce energy cost during sink-node communication [18] [31] [28], which is also the goal of our approach. However, nearly none of previous works have study on reducing energy cost of carrier sense due to the reason that carrier sense is intend to reduce energy.

Since the main point we focus on is the sink-node communication. More precisely, we need to implement CSMA/SF into the communication between sink and the ring of nodes around it (i.e. ring 1 in Fig.2(a)) to mitigate the "energy hole problem". To simplify this process, here we ignore the outer ring's routing effect and the nodes' distribution of different rings. In other word, the routing protocol and whether the distribution of sensor nodes in different rings is uniform or not is beyond our concern. However, since CSMA/SF does not restrict MAC protocol of outer-ring sensor nodes, it can coexists with CSMA/CA which can be used for nodes in outer rings.

The reason why we can ignore distribution and routing protocol of sensor nodes among different coronas is because that no matter what distribution or routing protocol we use in the WSN, the last process is always the communication of ring 1 nodes to sink. The effect of distribution and routing protocol of outer rings' nodes can only determine the workload of nodes in ring 1 . Given this into consideration, we can discuss different payload within ring 1 , which can be regard as the nodes' effect of outside rings. To sum up, the basic network 
model we mainly discuss is a 1 ring of sensor nodes with a center sink, which is shown in Fig.2(b).

\section{A2. Basic Assumptions in CSMA/SF}

Here are some basic assumptions in CSMA/SF model. We assume that all sensor nodes are deployed in a circular area. And all the sensor nodes are homogeneous. During the data transmission, all nodes can directly reach the sink. For the sake of simplicity, we assume the network is well connected. Each sensor node has a unique ID number. We assume that nodes in the inner side corona (e.g. ring1 in Fig.2(b)) is randomly distributed. All nodes in ring1 share a single radio channel for contention. And the channel access contention is a per packet process, which is that common CSMA/CA protocol does.

Another claim is that, there may exist hidden terminal problem within the inner side corona (e.g. ring1 in Fig.2(b)). Nevertheless, in our simplified model, since all the nodes are directly transmitting data to sink, there is no relay or other issue can cause expose terminal problem. As a potential assumption, there is no exposed terminal problem in our CSMA/SF model.

\section{B. Distributed Shortest-First Scheduling Algorithm}

Here we mainly discuss about our shortest-first scheduling algorithm. Different from traditional shortest-first scheduling algorithm which needs a centralized coordinator, our scheduling algorithm is totally distributed.

The key idea of our distributed shortest-first scheduling algorithm is based on the remaining data information of current transmitting node. Other nodes listen to this information and decide whether they have less data to transmit than current transmitting node. If some of them have less remaining data, they contend for channel access. Otherwise, they let the current transmitting node finish its transmission. The pseudo-code of distributed shortest-first scheduling algorithm is delivered as Algorithm 1.

In algorithm 1, listen_length is remaining data lengthen of current transmitting node, which is listened by the sensing node. data_length is the remaining data length of the listening node itself. Packet $v$ is the instantaneous packet that the transmitting node transmits, whereas packet $u$ is the newly channel-accessed node's first remaining packet.

As the example in Fig.1, since the first collision (i.e. time slot 2) happens during node2's transmission, node2 knows that the collided node must remain less packets to transmit. Thus, node2 will not try to contend for channel access after this collision. Instead, it turn into listening mode. And since node1 senses that channel is idle, it transmit its packet and turn into sleeping mode. After that, node2 senses channel is idle and finish its transmission.

Another part worth mentioning is procedure 18-25, which is the function that is used for avoiding contention collision. More precisely, it considers about the scenario that multinodes which remain less data to transmit than current transmitting node and contend for channel access. The function of this part pseudo-code also utilize traditional CSMA/CA's randomly back-off mechanism in order to reduce the collision probability of re-transmission packet. Indeed, CSMA/SF cannot completely be realized without CSMA/CA's randomly

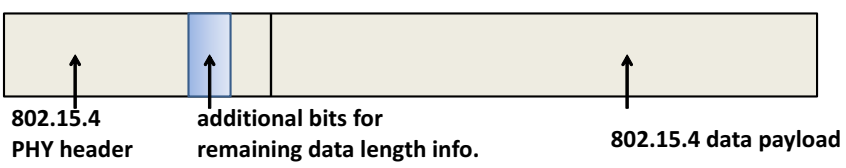

(a) Data length detection in slotted MAC protocol.

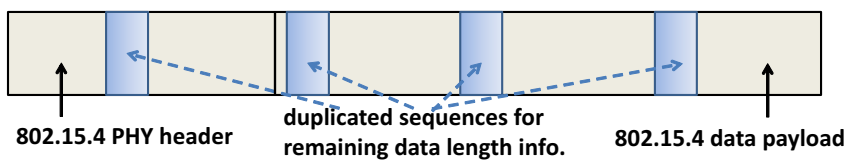

(b) Data length detection in unslotted MAC protocol.

Fig. 3. Data length detection scheme in slotted and unslotted MAC scenarios.

back-off mechanism. It can only reduce the total random backoff time to improve channel utilization.

However, this distributed scheduling algorithm cannot directly be implemented into real-world system. The main reason is that we cannot get the remaining data length of current transmitting node (i.e. listen_length). And this problem can be solved by incorporating Length Detection scheme to get the information of current transmitting node's remaining data length (i.e. listen_length).

\section{Length Detection Scheme}

How to get the remaining data length of current transmitting node is a key component of CSMA/SF. To achieve this goal, CSMA/SF adopts the Length Detection scheme. The Length Detection leverages that within the same band (e.g. $2.4 \mathrm{GHz}$ ), nodes transmit data in one unique modulation scheme in 802.15.4 [13] protocol. And this transmission process is broadcast. So every node which can directly reach to current transmitting node can hear what it transmits.

In this part, we first separately describe about the detailed Length Detection scheme in two scenarios that Standard 802.15.4 [13] can support, namely, beacon mode and nonbeacon mode. Then we propose a solution for hidden terminal issue in our Length Detection scheme.

\section{C1. Beacon Mode and Non-beacon Mode}

Standard 802.15.4 [13] protocol supports two transmission modes, namely beacon-based and non-beacon mode. The beacons are used to synchronize attached devices and describe the inner structure of superframe. With the superframe, the sensor nodes can achieve a slotted CSMA channel access mechanism. The non-beacon mode is a much simpler scheme that can only be used for unslotted CSMA channel contention.

In a star-like wireless network, the beacon mode appears to be more energy efficient than non-beacon mode. In other words, the slotted CSMA/CA is more energy-efficient than unslotted CSMA/CA. The reason is twofold. First, the slotted CSMA/CA can achieve less collision probability than unslotted one [22] [14]. Second, slotted CSMA/CA protocol allows transceiver to be completely in sleeping mode for most of idle time [22]. So here we mainly focus on the sink-node communication with slotted CSMA/CA MAC protocol. And then we will deliver another shortest-first scheme which is specially designed for unslotted CSMA MAC protocol. 
For slotted MAC protocol modification, as shown in Fig.3(a), we only need to modify PHY (Physical Layer) header by adding additional bits that represents the length of data remains to be sent. And every time other listening nodes can get the remaining data information of current transmitting node by decoding the additional part of transmitted packet's PHY header. In fact, there is no need for us to know about specific remaining data length. For the scenario that data length of different nodes varied in a large range, we can set remaining data length to different level and use additional bits to represent these levels instead of specific data length. By doing this, we can reduce the length the added bits and make data length detection more efficient.

For unslotted MAC protocol modification, we need to reconstruct the whole data package by inserting duplicate version of one specific data sequence as illustrated in Fig.3(b). Recall that the data modulation is unique in one specific frequency band (e.g. in $2.4 \mathrm{GHz}$ band, modulation scheme is O-QPSK). Hence, the receiver can exploit it directly by highly self-correlation between these duplicated sequences. By implementing this inserting process, it cannot only reduce the time for other listening nodes to get transmission information of current transmitting node, but also reduce transmission error. Since we can set remaining data length level instead of detailed length information, this duplicated inserting process will not cause noticeable overhead.

The reason for inserting duplicate sequences that represent the length information of remaining data is twofold. First, by inserting duplicate sequences can improve efficiency of nodes' length detection process. In other words, if we only adding additional bits in PHY header, other nodes should listen to the channel for the whole packet to get the remaining length information in bad cases. As we use duplicate inserting, other nodes only need to listen to the channel for no more than the interval of two adjacent inserted sequences. Another benefit of this inserting duplicate data is that it can reduce transmission error. More precisely, after the receiver exploit the contention of these duplicated sequence, these sequences can be regarded as pilots which can apparently reduce the transmission error and be helpful for data recovery.

\section{C2. Solution for Hidden Terminal Problem}

On the other hand, another problem we need to solve is the hidden terminal issue. More precisely, for the nodes that cannot directly hear the transmission of current sending node, we need to design a scheme to solve this problem.

Generally, we also leverage the RTS/CTS technique to solve hidden terminal problem. However, by implementing this mechanism into CSMA/SF will introduce significant overhead. Here we also make a modification with traditional RTS/CTS scheme. More precisely, instead of using RTS/CTS mechanism all the time, we use it only when collision caused by hidden terminal happened. Then the sink and collision nodes can use this RTS/CTS mechanism to coordinate the hidden nodes' transmission.

How to determine one specific kind of collision is caused by hidden terminal problem is a key issue we need to solve. Here we intuitively determine this problem by the times of one specific collision happens. To be more specific, if the collision continuously happened during one specific period, we predict that it may have high probability that is caused by hidden terminal problem. And then we enable RTS/CTS mechanism to solve it. On the other hand, even the continuously happened collision is not caused by hidden terminal problem, by enabling RTS/CTS will also reduce the probability of collision during transmission.

\section{Anti-Starvation Mechanism}

In any shortest-first scheduling algorithm implementations, there will exist starvation problem. To be more specific, there may be circumstances that the nodes with long data have no chance to transmit because there always exists short data transmission. Here the nodes with long data may get stuck and cannot transmit its data for very long time.

In order to solve this starvation problem, we add a periodical transmission mechanism to our CSMA/SF MAC protocol, which is called Anti-Starvation mechanism. The intuitive idea is to set a timeout scheme. When one node has not transmitted for a long time (i.e. longer than the timeout threshold), the timeout mechanism enabled and ensure this node to have highest priority in channel contention and be able to transmit some of its packets.

In Anti-Starvation mechanism, each node has a inner clock which keeps the upper bound of none transmission period. The clock decrease the counted time when node is not transmission and reset to the upper bound when node get a chance to transmit. When the clock is timeout (i.e. decrease counted time to zero), it modify remaining length information of a bunch of packets to 0 and contend for media access. After getting access to the channel, it transmits this bunch of packets directly. How large is the this modified quantity of packets is depend on the system's requirements. More precisely, if the system is delaytolerant, we can set this number small, otherwise set it large. The basic principle is that, the larger this number is, the less energy it saves in overall carrier sense process.

\section{E. Theoretical Performance Analysis of CSMA/SF}

In this part, we give a formal theoretical analysis about our proposed CSMA/SF MAC protocol. Our analysis main consists of two parts. The first one is energy saving on carrier sense process by implementing CSMA/SF. The second part is to briefly discuss about the channel utilization performance that CSMA/SF can achieve.

What we mainly focus on is the sink-node communication, which means the data transmission between the sink and the most inner ring (e.g. Ring1 in Fig.2(b)) of sensing nodes around it. In 802.15.4 standard [13]. As mentioned before, there are mainly two CSMA/CA protocols, namely, slotted and unslotted. In general, the slotted CSMA/CA is widely used in common WSNs [22]. Here we mainly discuss CSMA/SF based on the slotted scenario.

\section{E1. Energy Saving Analysis}

We now give a theoretical analysis about the nearly minimizing energy cost of carrier sense that CSMA/SF can achieve. As illustrated in Fig.2(b), the nodes in ring1 are randomly distributed. On average, per node traffic load in ring1 can be delivered as [15], which is as equation 1: 


$$
\begin{aligned}
\text { Load }_{\text {ring } 1} & =\frac{\text { total traffic in network }}{\text { No. of nodes in ringl }} \\
& =\frac{\rho(M r)^{2} b}{\rho \pi r^{2}}=\frac{M^{2}}{\pi} b
\end{aligned}
$$

In equation $1, \rho$ is the average density of nodes in ring 1 . $M$ is the number of rings the whole WSNs is divided (e.g. in Fig.2(a), M=3). $r$ is the radius of ring1. And $b$ is the transmission bitrate.

Since the nodes in ring 1 are randomly distributed, we assume for each node $i$, its real work load is $L_{i}$. And there are $\mathrm{N}$ nodes in ring1 in total. And overall workload of these $\mathrm{N}$ nodes in ring1 must be restricted to $N \frac{M^{2}}{\pi} b$.

In WSNs, there is no difference among the energy levels dissipated during sensing, transmission or reception [31]. To simplify this process, we assume the energy dissipation of carrier sense $E_{c s}$ is proportional to the time of nodes in active mode for this process $T_{a c t}(C S)$, which is delivered as equation 2 .

$$
E_{c s} \propto T_{a c t}(C S)
$$

On the other hand, in per packet contention scenario, for each node, the times for carrier sense is roughly as many as the quantity of packets transmitted until this node finish its transmission. And for each round of carrier sense, the sensing time is fixed, namely $\alpha$. Given this, we can conclude that the time for carrier sense is proportional to the quantity of packets that one node need to listen before it finishing its transmission (i.e. $P_{\text {lis }}$ in equation 3.), as described in equation 3.

$$
T_{a c t}(C S)=\alpha P_{l i s}
$$

With equation 2 and 3, we can conclude that the energy consumed in carrier sense is proportional to the number of packets one node should listen to. As shown in equation 4, the proportion factor between $E_{c s}$ and $T_{a c t}(C S)$ is $\xi$,

$$
E_{c s}=\xi \alpha P_{l i s}
$$

With these basic equations, we can model our problem of minimizing energy cost in carrier sense as following equation,

$$
\begin{array}{ll} 
& \text { Min } \quad E_{c s}=\xi \alpha P_{l i s} \\
\text { s.t. } & \sum_{i=1}^{N} L_{i}=N \frac{M^{2}}{\pi} b \\
& P_{l i s}(i)=\sum_{j=1}^{N} P_{j i}+L_{i} \quad(i \neq j) \\
& P_{l i s}=\sum_{i=1}^{N} P_{l i s}(i)
\end{array}
$$

In the above functions, $P_{l i s}(i)$ is the overall times that node i need to listen and carrier sense to. It is equal to the number of packets that other nodes transmitted before the last packet transmission of node i (i.e. $\sum_{j=1}^{N} P_{j i}$ in equation 7) plus the workload of node i itself (i.e. $L_{i}$ in equation 7). And here the overall times of carrier sense is equal to $P_{l i s}$, which is the sum up of all nodes's carrier sense times. Based on these functions, we can get the optimal solution as equation 9, suppose there are $\mathrm{N}$ nodes in total.

$$
\begin{aligned}
\operatorname{Min} E_{c s} & =\xi \alpha P_{l i s} \\
& =\xi \alpha\left[N L s_{1}+(N-1) L s_{2}+\cdots+L s_{N}\right] \\
& =\xi \alpha\left[\sum_{i=1}^{N}(N-i+1) L s_{i}\right]
\end{aligned}
$$

In equation $9, L s_{i}$ is the sorted workload of nodes with ascending order. For example, $L s_{1}$ is the workload of the nodes with the smallest amount of data remaining to transmit. And $L s_{2}$ is the second smallest whereas $L s_{N}$ is the payload of nodes with largest amount of data to transmit.

Apparently the solution of this "Min $E_{c s}$ " problem is just approximate with the result of our shortest-first scheduling algorithm. More precisely, in order to achieve overall minimum times of carrier sense, the optimal solution is to firstly sort all nodes' workload from the smallest to the largest and then let them transmit to sink in this ascending order. If there is no collision, our CSMA/SF can achieve optimal result of minimizing the energy cost in carrier sense.

This mathematical model proves that our CSMA/SF can achieve the nearly optimal energy saving result of carrier sense process.

\section{E2. Channel Utilization Efficiency Analysis}

In this part, we give a theoretical analysis about how CSMA/SF can improve channel utilization compared with traditional CSMA/CA MAC protocol.

$$
\begin{aligned}
\varepsilon_{p} & =N p_{1}\left(1-p_{1}\right)^{N-1}+N p_{2}\left(1-p_{1}-p_{2}\right)^{N-1} \\
& +\cdots+N p_{M}\left(1-p_{1}-p_{2} \cdots-p_{M}\right)^{N-1} \\
& =N \sum_{k=1}^{M} p_{k}\left(1-\sum_{x=1}^{k} p_{x}\right)^{N-1}
\end{aligned}
$$

Suppose there are $M$ slots and there are $N$ nodes contend for the channel access independently. And let $k$ be the slot number. For a slot $\mathrm{x}$, each node pick it with a probability of $p_{x}$. And the probability distribution of this slot picking process (i.e. $p_{1}, p_{2}, \ldots p_{M}$ ) can be any kind. Here we refer this distribution as $p$.

Let $\varepsilon_{p}$ be the probability of all $N$ nodes successfully picking a slot for contention under the probability distribution $p$ of this picking process. To make it more clear, we illustrate the first part of equation 10 (i.e. $\left.N * p_{1} *\left(1-p_{1}\right)^{N-1}\right)$. Here $p_{1} *\left(1-p_{1}\right)^{N-1}$ means one specific node successfully picking slot 1 (i.e. $p_{1}$ )while other $\mathrm{n}-1$ nodes not picking slot 1 (i.e. $\left.\left(1-p_{1}\right)^{N-1}\right)$. Since all nodes share the same successful pick probability, for any one node, we should multiply the probability of successfully picking slot 1 with the quantity of nodes (i.e. $N$ ). So the overall probability of successfully transmission probability is $N * p_{1} *\left(1-p_{1}\right)^{N-1}$. And the representation for the success transmission probability of time slot 2,3...M is similar to slot 1 . Based on this definition, we can get the probability of success $\varepsilon_{p}$ is the sum of the success probability in each time slot before slot $M+1$, which is as the equation 10 . 


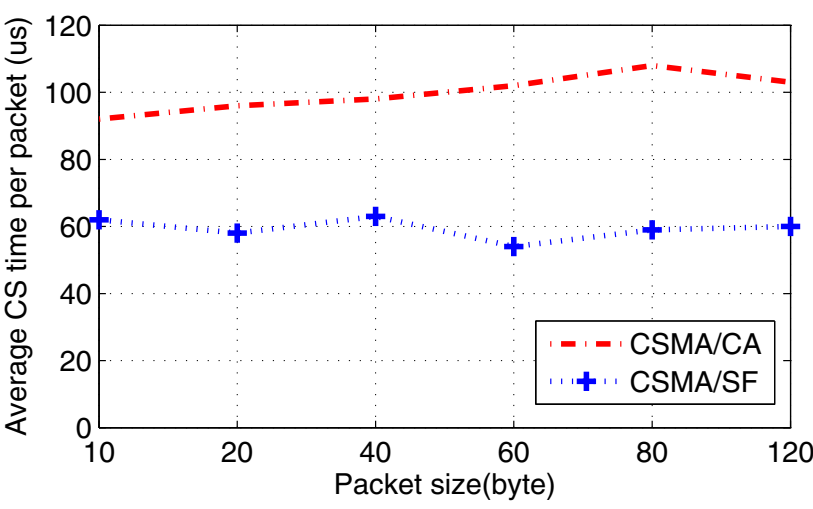

Fig. 4. Energy consumption comparison between slotted CSMA/CA and CSMA/SF.

By implementing CSMA/SF, we modify CSMA/CA's completely contention-based channel access protocol with additional priority. Since nodes can know the remaining data length of current transmitting node, the nodes will not content for channel access if they remain more data than current transmitting one. Intuitively, by implementing CSMA/SF, we reduce the number of nodes content in each time slot.

From the view of $\varepsilon_{p}$ in equation 10, we reduce the number of exponent value $N-1$. Since $1-\sum_{x=1}^{k} p_{x}$ is less than 1 , with the decreasing of exponent $N-1$, it apparently increase the overall success probability $\varepsilon_{p}$. Thus by implementing CSMA/SF, compared with traditional CSMA/CA, it improves the overall channel utilization.

\section{CSMA/SF PROTOCOL IMPLEMENTATION}

In this section, we present detailed implementation of CSMA/SF MAC protocol with its assistant Anti-Starvation and Length Detection schemes. Due to the limited number of pages, we only describe the key components and most challenging issues of the implementing process.

The experimental software platform is GNU Radio [1]. The IEEE 802.15.4 protocol we applied as the base scheme is one mature PHY implementation [24]. We employ it on USRP2 platform [7]. Based on this prototype, we modified its MAC layer and PHY layer for implementation of CSMA/SF. For large scale simulation, we use NS2 [20] as the simulation platform.

\section{A. Length Detection Scheme Implementation}

In this part, we deliver the implementation of Length Detection Scheme. We mainly focus on slotted situation since it is more efficient. After that we will briefly discuss about the design scheme of implementing Length Detection in unslotted scenario.

In IEEE 802.15.4 [13] standard, the traditional PPDU (Physical Protocol Data Unit) packet format consists of three parts, namely synchronization header (SHR), PHY header (PHR) and PHY payload. In order to adding remaining data length information on the PHR, we need to extend its size. The initial length of PHR is 8 bits which contains 7 bits representing frame length and 1 bit reserved.

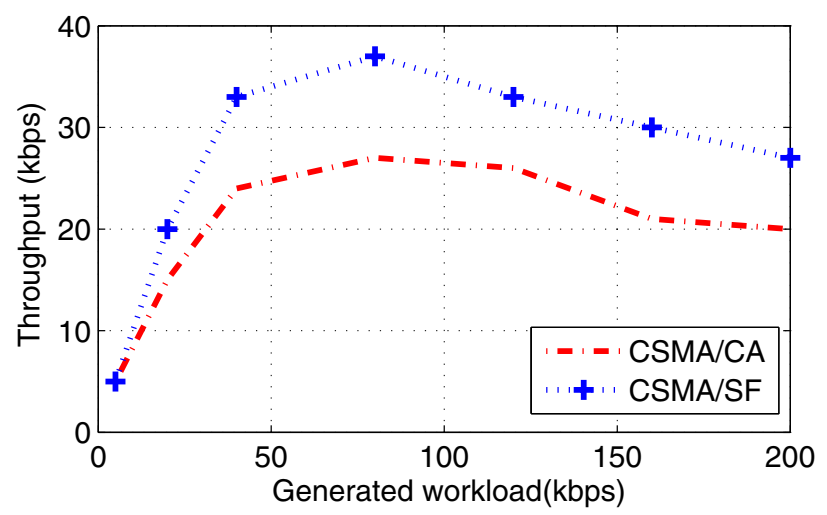

Fig. 5. Throughput comparison between slotted CSMA/CA and CSMA/SF.

For reducing the overhead of these additional bits, here we use these bits only represent the remaining data length levels instead of specific length. With 1 bit reserved, we add another 5 bits. Thus the total amount of bits are 6 , which can represent data length with $2^{6}$ different levels.

For the unslotted scenario, the implementation is more challenging. There are mainly two difficulties. First, since we want to insert duplicated data length information throughout the whole packet, we must reduce the overall overhead of this inserting process. Second, in unslotted scenario, since the sensing nodes cannot synchronize with transmitting node, they cannot directly decode the duplicated sequences. Here we propose a special PN sequence design. In stead of inserting remaining data length information like original data coding process, we generate special PN sequence (like Gold sequence [8]) which the sequence's length conveys the remaining data length information. So here for carrier sensing nodes, they only need listen to this specialized PN sequence to know the remaining data length of current transmitting node.

\section{B. Anti-Starvation Mechanism Implementation}

In this part, we mainly talk about how to deploy AntiStarvation Mechanism. The key issue here is to determine the timeout's upper bound and the quantity of periodic transmitted packets.

Generally, the timeout value should take transmission rate and the number of sensor nodes into consideration. In IEEE 802.15 .4 [13] standard, the transmission rate is $250 \mathrm{~kb} / \mathrm{s}$ in 2.4 $\mathrm{GHz}$ band. Suppose the average packet size is 120 bytes and there are 100 nodes in ring 1 , we set the upper bound of timeout value to $400 \mathrm{~ms}$. It is the approximation of transmission time of 100 packets. And we set the amount of periodic transmitting packets to 10 .

\section{EXPERIMENTAL EVALUATION}

In this section, we mainly describe and evaluate about slotted CSMA/SF's performance, which compared with traditional CSMA/CA. There are mainly two periods. First, we deploy CSMA/SF on USRP2 [7] to verify small scale performance. After that, we simulate CSMA/SF on NS2 [20] simulator to analyze the energy efficiency and channel utilization performance for large scale WSNs (with up to 100 nodes in inner side ring as ring1 in Fig.2(b)). 

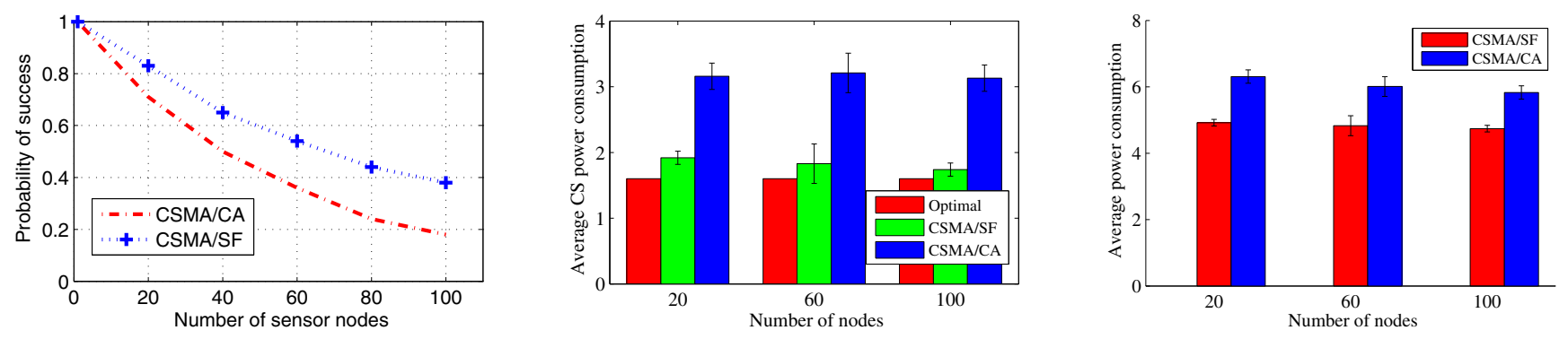

Fig. 6. Probability of success access comparison Fig. 7. Carrier sense energy consumption of Fig. 8. CSMA/SF overall energy cost. between slotted CSMA/CA and CSMA/SF. CSMA/SF.

\section{A. Performance Analysis on USRP2}

In this part, we evaluate the performance of CSMA/SF on USRP2. For the energy cost measurement, we regard overall carrier sense time as the corresponding energy consumption. It is because the proportion relationship between carrier sense time and its corresponding energy consumption that we have proved in section 3 . Here we use 2 USRP2 nodes as senders and one USRP as the receiver (i.e. sink). We generate the data payload randomly from 0 byte to 200 bytes for each sender in 10 transmission rounds. After that we refresh the buffer and generate workload for a new round. And we use varied packet size as shown in Fig.4. We empirically set timeout upper bound of our Anti-Starvation mechanism to $20 \mathrm{~ms}$. And we set the corresponding quantity of periodic transmitting packets to 2.

The remaining data length is randomly generated by a simplified Markov chain model. If the Markov chain generates data, the sender will carrier sense and then decide when to begin the transmission. And the dynamically generated data will be represented as the remaining data length of the sender. Here we have the idle sleeping mode on, which has been implemented in the 802.15.4 prototype [24] we used.

Here we use the corresponding carrier sense (CS) time as the energy cost. It is because we have proved in Section 3 that CS time is proportional to its corresponding energy cost in WSNs. As shown in Fig.4, the corresponding (CS) time of CSMA/SF is much less than traditional CSMA/CA. The reduction of $\mathrm{CS}$ energy cost is ranging from $30 \%$ to $40 \%$, compared with CSMA/CA. And it seems that the CS time remains nearly the same with varied packet size in both CSMA/CA and CSMA/SF.

On the channel utilization part, Fig.5 shows that CSMA/SF apparently improve throughput up to $40 \%$ compared with traditional CSMA/CA MAC protocol. More precisely, with the workload increasing, CSMA/SF can maintain a higher level throughput than CSMA/CA. The reason why throughput has a bottleneck around $42 \mathrm{kbps}$ may because the control overhead of beacon or superframe packets. As a whole, CSMA/SF outperforms CSMA/CA by $34 \%$ on average in different workload scenarios.

\section{B. Performance Evaluation on NS2}

In this part, we evaluate the performance of CSMA/SF in NS2 simulation. We mainly focus on energy saving and channel utilization. The parameters we used for our model is illustrated as follows. For power state, the receiving and carrier sensing is equal to $40 \mathrm{~mW}$, whereas transmitting costs $30 \mathrm{~mW}$. Idle listening consumes $0.8 \mathrm{mw}$ and sleep costs $0.1 \mu \mathrm{W}$. The packet format and transmission parameters we use are standard IEEE 802.15 .4 [13] for $2.4 \mathrm{GHz}$ band. We generate the data payload randomly from 0 byte to 200 bytes for each node in a $\mathbf{M}$ time slot round. Here $\mathbf{M}$ is the same as it in equation 10 . And we set $M$ equal to the quantity of nodes. We empirically set timeout upper bound of our Anti-Starvation mechanism to $80 \mathrm{~ms}, 240 \mathrm{~ms}, 400 \mathrm{~ms}$ for $20,60,100$ nodes contention scenarios respectively. And this timeout upper bound setting method has been described in Section IV-B. And we set the amount of periodic transmitting packets to $2,6,10$ respectively for 20, 60, 100 nodes scenarios.

As shown in Fig.6, the simulation result validates our proof that the probability of success accessing $\varepsilon_{p}$ can be improved by implementing CSMA/SF MAC protocol. To be more specific, with the number of nodes increasing, the residual between CSMA/SF and CSMA/CA became larger, which indicates that CSMA/SF can have better channel utilization than CSMA/CA. And Fig.9, 10, 11 give a more clear result that CSMA/SF achieves better channel utilization by improving throughput with different amount of nodes (Namely 20, 60, 100 nodes). More precisely, with the nodes number increasing, the difference between CSMA/SF and CSMA/CA become smaller. It is because that, with more nodes contending for channel access, nodes with less data try to occupy channel by interrupting current transmission node. And this increasing number of interruption will cause more collisions. Even though CSMA/SF can reduce the number of contention nodes in any specific time slot, it cannot improve channel utilization with large number of nodes. It is because the increasing amount of collisions caused by interruption cancel-out with the reduction of contention collisions that CSMA/SF achieves.

Fig.7 shows the result of energy saving that CSMA/SF can achieve with different amount of nodes. To be more specific, with the simulation result, CSMA/SF can reduce the energy cost on carrier sense by about $37 \%$ on average. And CSMA/SF can nearly achieve optimal energy cost of CS in different scenarios. The difference between optimal energy cost and CSMA/SF is mainly because the transmission in optimal scenario is strictly in ascending order of remaining data length. As in CSMA/SF, we cannot achieve the theoretical optimal because of randomly generated data length. In other words, there may exist nodes with shorter data than current node that with the shortest remaining data length in the following time 


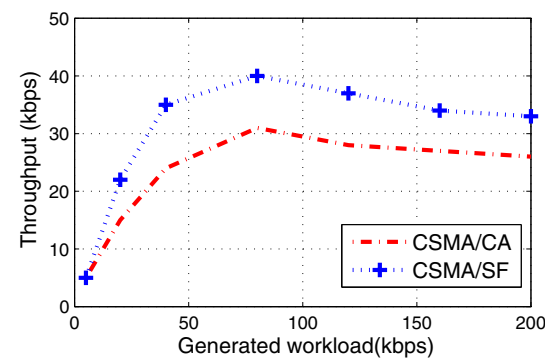

Fig. 9. CSMA/SF throughput performance with 20 Fig. 10. nodes.

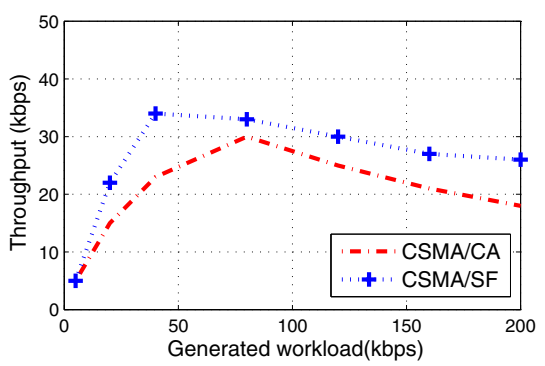

60 nodes.

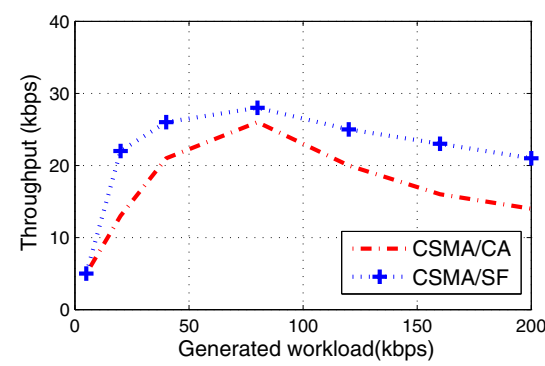

Fig. 11. CSMA/SF throughput performance with 100 nodes. slots.

For the total energy cost calculated in Fig.8, CSMA/SF can save around $20 \%$ of nodes' total energy cost. It is because the fact that, the proportion of CS energy cost will be nearly $50 \%$ of overall energy cost with nodes number larger than 10 . And previous paper [22] also verifies this point. From the result of energy cost in our simulation, it is verified that carrier sense is really a large energy consumption component which cannot be negligible in large scale WSNs.

To sum up, as both USRP2 experiment and NS2 simulation results show that, compared with CSMA/CA, by implementing CSMA/SF can reduce nealy $37 \%$ energy cost on CS and achieve overall energy saving around $20 \%$. Additionally, since with contention priority, CSMA/SF can also improve channel utilization up to $40 \%$ compared with traditional CSMA/CA.

\section{CONCLUSIONS}

This paper proposes a new MAC protocol CSMA/SF to minimize energy cost in carrier sense process. By modifying existed purely contention-based CSMA/CA with additional priority, we ensure the nodes remain less data has higher priority in channel access contention. To achieve this, we design mainly three components, namely, a distributed shortestfirst algorithm, Length Detection scheme and Anti-Starvation mechanism. The distributed shortest-first algorithm is used for achieving shortest-first scheduling process in a distributed way. We incorporate Length Detection scheme to let the listening nodes get the remaining length information of current transmitting node. And Anti-Starvation mechanism is used for addressing the starvation problem that shortest-first algorithm will cause. With theoretical analysis and experimental evaluation, our approach outperforms existed CSMA/CA by reducing overall energy cost around $20 \%$ and improving channel utilization up to $40 \%$. Since CSMA/SF follows traditional MAC protocol design and is very easy to be realized, we believe CSMA/SF can be beneficial to real world applications.

\section{ACKNOWLEDGEMENT}

This research is supported in part by Program for New Century Excellent Talents in University (NCET-130908), Guangdong Natural Science Funds for Distinguished Young Scholar (No.S20120011468), Hong Kong RGC Grant HKUST617212, New Star of Pearl River on Science and Technology of Guangzhou (No.2012J2200081), Guangdong NSF Grant (No.S2012010010427), China NSFC Grant 61202454. Kaishun $\mathrm{Wu}$ is the corresponding author.

\section{REFERENCES}

[1] E. Blossom, "GNU software defined radio." Available: http://www.gnu. org/software/gnuradio.

[2] M. Z. Brodsky and R. T. Morris, "In defense of wireless carrier sense," in Proc. 2009 ACM SIGCOMM.

[3] J.-H. Chang and L. Tassiulas, "Routing for maximum system lifetime in wireless ad-hoc networks," in Proc. 1999 Allertion Conf. Commun., Control, Comput.

[4] - "Maximum lifetime routing in wireless sensor networks," IEEE/ACM Trans. Netw., vol. 12, no. 4, 2004.

[5] S. C. Ergen and P. Varaiya, "Quality-of-service in ad hoc carrier sense multiple access wireless networks," IEEE J. Sel. Areas Commun., vol. 17 , no. 8, 1999.

[6] — - "Pedamacs: power efficient and delay aware medium access protocol for sensor networks," IEEE Trans. Mobile Comput., vol. 5, no. 7, 2006.

[7] Ettus_Research_LLC, Universal Software Radio Peripheral. Available: http://www.ettus.com.

[8] P. Fan and M. Darnell, Sequence Design for Communications Applications. Research Study Press, 1996.

[9] S. R. Gandham, M. Dawande, R. Prakash, and S. Venkatesan, "Energy efficient schemes for wireless sensor networks with multiple mobile base stations," in Proc. 2003 IEEE GLOBECOM.

[10] M. Gatzianas and L. Georgiadis, "A distributed algorithm for maximum lifetime routing in sensor networks with mobile sink," IEEE Trans. Wireless Commun., vol. 7, no. 3, 2008.

[11] Y. Gu, Y. Ji, J. Li, B. Han, and B. Zhao, "Delay-bounded sink mobility in wireless sensor networks," in Proc. 2012 IEEE ICC.

[12] E. Haghani, M. N. Krishnan, and A. Zakhor, "Adaptive carrier-sensing for throughput improvement in IEEE 802.11 networks," in Proc. 2010 IEEE GLOBECOM.

[13] IEEE_Standard802.15.4, IEEE Standard 802.15.4: Wireless Medium Access Control (MAC) and Physical Layer (PHY) Specifications for Low-Rate Wireless Personal Area Networks (WPANs). Available: http: //standards.ieee.org/getieee802/download/802.15.4-2006.pdf.

[14] T. O. Kim, J. S. Park, H. J. Chong, K. J. Kim, and B. D. Choi, "Performance analysis of IEEE 802.15.4 non-beacon mode with the unslotted csma/ca," IEEE Commun. Lett., vol. 12, no. 4, 2008.

[15] J. Li and P. Mohapatra, "An analytical model for the energy hole problem in many-to-one sensor networks," in Proc. 2005 Veh. Technol. Conf.

[16] _ - "Analytical modeling and mitigation techniques for the energy hole problem in sensor networks," Pervasive Mobile Comput., vol. 3, no. 3, 2007.

[17] Z. Li, M. Li, and Y. Liu, "Towards energy-fairness in asynchronous duty-cycling sensor networks," in Proc. 2012 INFOCOM.

[18] J. Ma, W. Lou, Y. Wu, X.-Y. Li, and G. Chen, "Energy efficient TDMA sleep scheduling in wireless sensor networks," in Proc. 2009 IEEE INFOCOM.

[19] M. J. Miller and N. H. Vaidya, "Improving power save protocols using carrier sensing for dynamic advertisement windows," in Proc. 2005 IEEE MASS.

[20] ns2 simulator, Network Simulator. Available: http://www.isi.edu/nsnam/ $\mathrm{ns} /$.

[21] S. Olariu and I. Stojmenovic, "Design guidelines for maximizing lifetime and avoiding energy holes in sensor networks with uniform distribution and uniform reporting," in Proc. 2006 IEEE INFOCOM.

[22] S. Pollin, M. Ergen, S. C. Ergen, and B. Bougard, "Performance analysis of slotted carrier sense IEEE 802.15.4 medium access layer," IEEE Trans. Wireless Commun., vol. 7, no. 9, 2008. 
[23] L. Popa, A. Rostamizadeh, R. M. Karp, C. Papadimitriou, and I. Stoica, "Balancing traffic load in wireless networks with curveball routing," in Proc. 2007 ACM MobiHoc.

[24] T. Schmid, "GNU radio 802.15.4 en- and decoding," UCLA NESL Tech. Rep., 2005.

[25] Y. Shi and Y. T. Hou, "Theoretical results on base station movement problem for sensor network," in Proc. 2008 IEEE INFOCOM.

[26] L. Tang, Y. Sun, O. Gurewitz, and D. B.Johnson, "PW-MAC: an energyefficient predictive-wakeup MAC protocol for wireless sensor networks," in Proc. 2011 IEEE INFOCOM.

[27] Y. C. Tay, K. Jamieson, and H. Balakrishnan, "Collision-minimizing CSMA and its applications to wireless sensor networks," IEEE J. Sel. Areas Commun., vol. 22, no. 6, 2004.

[28] T. van Dam and K. Langendoen, "An adaptive energy-efficient MAC protocol for wireless sensor networks," in Proc. 2003 ACM SenSys.

[29] X. Wu, G. Chen, and S. K. Das, "Avoiding energy holes in wireless sensor networks with nonuniform node distribution," IEEE Trans. Parallel Distributed Syst., vol. 19, no. 5, 2008.

[30] W. Ye and J. Heidemann, "Medium access control in wireless sensor networks," USC/ISI Tech. Rep.

[31] W. Ye, J. Heidemann, and D. Estrin, "An energy-efficient MAC protocol for wireless sensor networks," in Proc. 2002 IEEE INFOCOM.

[32] Y. Yun and Y. Xia, "Maximizing the lifetime of wireless sensor networks with mobile sink in delay-tolerant applications," IEEE Trans. Mobile Comput., vol. 9, no. 9, 2010.

[33] Z. Zeng, Y. Yang, and J. C. Hou, "How physical carrier sense affects system throughput in IEEE 802.11 wireless networks," in Proc. 2008 IEEE INFOCOM.

[34] M. Zhao and Y. Yang, "Bounded relay hop mobile data gathering in wireless sensor networks," IEEE Trans. Comput., vol. 61, no. 2, 2012.

[35] Y. Zhu and L. M. Ni, "Probabilistic wakeup: adaptive duty cycling for energy-efficient event detection," in Proc. 2007 ACM/IEEE MSWiM.

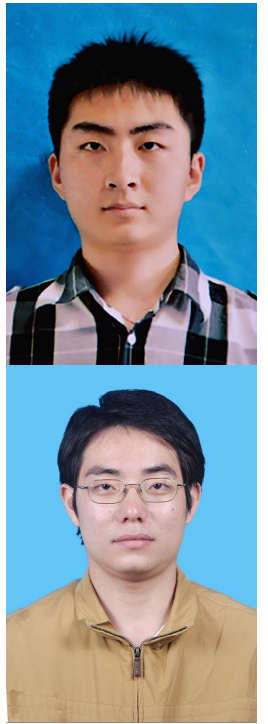

Guanhua Wang received his B.Eng. degree from Southeast University, Nanjing, China, in 2012, and is currently working towards the Ph.D. degree in computer science and engineering in Hong Kong University of Science and Technology. His main research interests include wireless communication, mobile computing and wireless sensor networks.

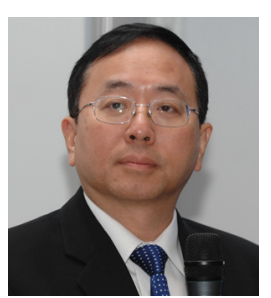

Lionel M. Ni is Chair Professor in the Department of Computer Science and Engineering at The Hong Kong University of Science and Technology (HKUST). He also serves as the Special Assistant to the President of HKUST, Dean of HKUST Fok Ying Tung Graduate School and Visiting Chair Professor of Shanghai Key Lab of Scalable Computing and Systems at Shanghai Jiao Tong University. A fellow of IEEE, Dr. Ni has chaired over 30 professional conferences. 